\title{
Ein Achteljahrtausend DMV und andere Jubiläen
}

Peter Ullrich

Am 18. September 1890 unterzeichneten 33 Mathematiker, die in Bremen im Rahmen der 63. Jahresversammlung der Gesellschaft Deutscher Naturforscher und Ärzte (GDNÄ) zusammengekommen waren, einen Aufruf zur Gründung einer Vereinigung deutscher Mathematiker. Da bei dieser Gelegenheit bereits ein Vorstand gewählt und 1891 in Halle die erste Jahresversammlung der dort so getauften Deutschen Mathematiker-Vereinigung (DMV) abgehalten wurde, wird das genannte Datum als der Geburtstag der DMV angesehen. Nachdem es im Laufe des 19. Jahrhunderts mehrfach Anläufe zu einer Fachvereinigung der deutschen Mathematiker gegeben hatte, war jetzt Georg Cantor (1845-1918) erfolgreich gewesen, unter anderem unterstützt von Walter (später: von) Dyck (1856-1934) und damit, im Hintergrund, von Felix Klein (1849-1925). Cantor wurde der erste Vorsitzende, Dyck der erste Schriftführer der DMV.

Somit konnte die DMV letztes Jahr ihr I25-jähriges Bestehen feiern. Hieran ist durch ihren Präsidenten bereits in den Mitteilungen erinnert worden; weiterhin veröffentlichte Renate Tobies im Programmbuch der Hamburger Jahrestagung 2015 einen wissenschaftlichen Artikel mit dem Titel „,I25 Jahre DMV“ [9].

Sowohl die Geschichte der DMV im Allgemeinen als auch die ihrer Gründung im Speziellen ist ausführlich dokumentiert und intensiv erforscht worden: Die Website [12] führt einschlägige Literatur auf; der letzte Artikel zu dem Themenkreis in den Mitteilungen stammt von Gert Schubring aus dem Jahr 2010 anlässlich des 120. Jubiläums der DMV [8].

Allerdings ist die DMV nicht alleine mit einem durch 25 teilbaren Jahrestag: Die European Mathematical Society (EMS) wurde 2015 zwar „nur“ 25 Jahre alt, aber, wie ihr Präsident Pavel Exner in seiner aktuellen „Message from the President" [5] anmerkt:

The year that just ended was marked by numerous jubilees. It was not only the Society 25th birthday [...]. In a remarkable harmony some of our corporate members celebrated jubilees marked by integer multiples of the EMS's age - six for the London Mathematical Society, five for Deutsche Mathematiker-Vereinigung, and three for Sociedade Portuguesa de Matemática.

Diese Beispiele scheinen zu belegen, was man für den Gründungszeitpunkt wissenschaftlicher Fachgesellschaften zur Mathematik vielleicht erwarten würde:

- Sehr alt sind die Gesellschaften in Staaten, die sich bereits Anfang des 19. Jahrhunderts als politisches Gebilde konsolidiert hatten und die bereits in jener Zeit
Zentren der mathematischen Forschung beherbergten, sei es, dass sie wie die 1865 gegründete London Mathematical Society (LMS) deren Hauptstadt im Namen tragen, sei es, dass sie nach dem Land benannt sind wie die 1872 gegründete Société Mathématique de France (SMF).

- In den anderen bereits frühzeitig konsolidierten Staaten gibt das Gründungsjahr ein Maß für den Grad der Etablierung der Mathematik als Fachdisziplin, insbesondere im Bereich der Hochschulbildung.

- In den sich erst im Laufe des 19. Jahrhunderts in Staatsgebilden konsolidierenden Nationen entstanden die Gesellschaften mit einer gewissen Verzögerung, so in Deutschland, aber auch in Italien, wo die Unione Matematica Italiana (UMI) erst 1922 gegründet wurde.

- Die Gründung von internationalen Vereinigungen erfolgte häufig im Anschluss an Veränderungen der politischen Großwetterlage: Nach durchaus längerer Vorbereitung entstand die EMS am Ende des Kalten Krieges, während die beiden Gründungen der International Mathematical Union (IMU) 1920 und 1950 in die Nachkriegszeit von Erstem bzw. Zweitem Weltkrieg fielen, vgl. [13].

Der Vergleich der Gründung der DMV mit der anderer Fachgesellschaften ist bislang nur exemplarisch geführt worden, siehe etwa [6] für den Vergleich von DMV und SMF, auch die Bemerkung in [8, S. 103]. So erweisen sich denn bei genauerer Betrachtung die obigen Thesen keinesfalls als Sätze mit Allgemeingültigkeit, sondern, um mit Niels Henrik Abel (1802-1829) zu sprechen, man stellt fest, dass so mancher „Lehrsatz Ausnahmen leidet“ - zu denen bis zu einem gewissen Grad auch die DMV selbst gehört.

Lokal, regional, national

Zunächst einmal wurde oben mit Absicht von ,sehr alten“ Gesellschaften geschrieben: Die nationale mathematische Gesellschaft mit den ältesten Wurzeln ist die Koninklijk Wiskundig Genootschap (KWG) der Niederlande. Den Zusatz „Koninklijk“ im Namen erhielt sie zwar erst 2003, gegründet wurde sie jedoch bereits 1778. Allerdings handelte es sich dabei zunächst um eine Vereinigung, deren Wirkung auf Amsterdam beschränkt war und die auch erst in der zweiten Hälfte des 19. Jahrhunderts aktiv zur mathematischen Forschung beitrug, vgl. [I], [2].

Das bekannteste und wohl auch schnellste Beispiel einer ähnlichen Ausdehnung von der lokalen Ebene auf ein ganzes Staatsgebilde ist die am 24. November 1888 von 


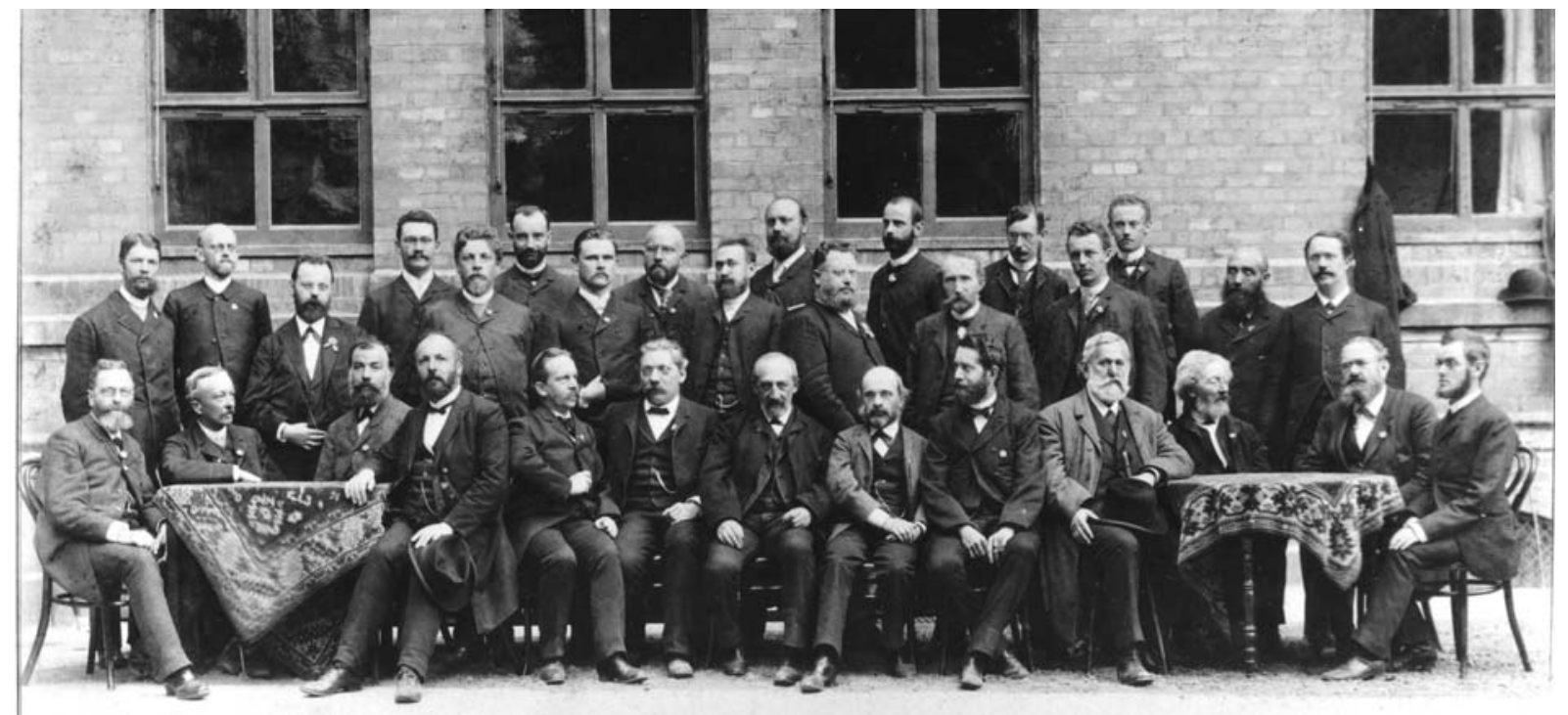

DMV-Gründungsgremium: Die Abteilung I der GdNÄ im September 1890 in Bremen

(Foto: J. Ortgies jr.; vgl. [I2])

sechs Mitgliedern der Columbia University gegründete New York Mathematical Society; deren Wirkungsbereich hatte sich in kürzester Zeit so erweitert, dass sie bereits im Frühjahr 1894 in American Mathematical Society (AMS) unbenannt wurde, was von ihr als Gründungsdatum betrachtet wird.

Räumlich näher liegt der Fall Österreichs: 1903 wurde eine Mathematische Gesellschaft in Wien gegründet und nach dem Zweiten Weltkrieg auch unter dem gleichen Namen am 10. August 1946 wiedergegründet. Erst 1948 fand die Umbennennung dieser Vereinigung in Österreichische Mathematische Gesellschaft (ÖMG) statt.

Insoweit ist es ein zwar müßiges, aber nicht völlig abwegiges Gedankenspiel, sich vorzustellen, die DMV hätte 1890 bei ihrer Gründung auf die Geschichte der damals gerade 200 Jahre alt werdenden Mathematischen Gesellschaft in Hamburg (MGH) zurückgegriffen und müsste sich demgemäß jetzt Gedanken wegen ihres Jubiläums zum Dritteljahrtausend im Jahre 2023 machen.

Als im Jahre 1690 die Kunst-Rechnungs-liebende Societät in Hamburg gegründet wurde, war die Royal Society in London zwar schon 30 Jahre alt. Da sich diese aber den $\mathrm{Na}$ turwissenschaften widmet, handelt es sich bei der $\mathrm{MGH}$, die 1872 ihren jetzigen Namen erhielt, um die älteste noch bestehende wissenschaftliche Fachgesellschaft speziell für die Mathematik, wenn auch nicht mit nationalem Vertretungsanspruch wie die KWG, vgl. [3, S. 3]. Zur Geschichte der MGH vgl. die Website [14] sowie die dort angegebene Literatur. Die Mathematischen Gesellschaften in Berlin und Göttingen hingegen wurden erst nach der DMV gegründet, 190I bzw. 1892. Immerhin war Hermann Cäsar Hannibal Schubert (1848-1911) nicht nur in der MGH führend tätig, sondern auch in die Vorbereitung zu Gründung der DMV einbezogen; er wurde sogar in ihren ersten Vorstand gewählt. Allerdings verfügte Hamburg zu jener Zeit noch über keine Universität: Schubert war Gymnasialprofessor am Johanneum in Hamburg und demgemäß in der DMV hauptsächlich für die Einbeziehung von Gymnasiallehrkräften zuständig, vgl. [10].

Was bedeutet(e) „Deutsch“?

Heutzutage sieht man die DMV zumeist als die für die Bundesrepublik Deutschland ,zuständige“ mathematische Vereinigung an. Dies wird dadurch gestützt, dass am 8. Juni 1962, also zwar schon einige Jahre nach der deutschen Teilung, aber recht kurz nach dem Bau der Grenzsperranlagen der Deutschen Demokratischen Republik, die Mathematische Gesellschaft in der DDR (MGDDR) gegründet wurde, die dann nach dem Beitritt der DDR zur Bundesrepublik 1990 mit der DMV vereinigt wurde.

Jedoch lehrt das schon erwähnte Beispiel der ÖMG, dass Fachgesellschaften nicht zwingend der staatlichen Gliederung folgen müssen: Die DMV definierte sich in ihren Gründungsstatuten über den Begriff der „Nation“, aber dies bedeutete eben keine Einschränkung auf das Deutsche Kaiserreich. Bereits 1894 war die Jahrestagung der DMV in Wien, 1902 in Karlsbad (damals Königreich Böhmen, heute Karlovy Vary in Tschechien); zahlreiche weitere Jahrestagungen fanden an anderen Orten des Habsburgerreiches bzw. dessen nach dem Ersten Weltkrieg entstandenen Nachfolgestaaten statt. 
Hingegen fehlt vollständig der deutschsprachige Bereich der Schweiz. Dort wurde auch, sozusagen im Gegenzug, schon 1910 eine Schweizerische Mathematische Gesellschaft (SMG bzw. SMS für Société Mathématique Suisse) gegründet.

Operativ bedeutete „deutsch“ im Namen der DMV in ihren ersten Jahrzehnten also gerade jenes Gebiet, das im 19. Jahrhundert die eine Alternative für Bildung eines Staates deutscher Nation darstellte, die sogenannte "großdeutsche“ unter Einbeziehung der deutschsprachigen Gebiete des Habsburgerreiches. Hierin kann man durchaus die Übernahme der Tradition der GDNÄ sehen, die 1822 gegründet worden war, also zur Zeit des Deutschen Bundes, eines Staatenbundes, der das genannte Gebiet umfasste. (Zur Geschichte der GDNÄ vergleiche [4].)

Allerdings entsprach dies zum Zeitpunkt der Gründung der DMV gerade nicht der staatlichen Struktur. In den Kriegen von 1864 bis 1870/7I war nicht die ,großdeutsche“ Alternative realisiert worden, sondern die „kleindeutsche" ohne Österreich und mit der Führungsmacht Preußen. Dem Deutsch(-Österreichisch)en Krieg von 1866 - der in diesem Jahr genau I50 Jahre zurückliegt war zwar bald eine politische Wiederannäherung zwischen dem Deutschen Kaiserreich und dem Habsburgerreich gefolgt, die sich etwa in dem Dreikaiserabkommen von 1873 und, vor allen Dingen, dem Zweibundvertrag von 1879 niederschlug. Das war aber sozusagen nur die außenpolitische Aufarbeitung.

Nach dem Krieg von 1866 hatte das siegreiche Königreich Preußen von seinen Kriegsgegnern das Königreich Hannover sowie zahlreiche hessische Gebiete annektiert. Hierdurch erhielt es zum Beispiel formal gesehen seine erste Polytechnische Hochschule, die in Hannover, auch wenn die Vorbereitung zur 1870 erfolgten Eröffnung der Polytechnische Hochschule in Aachen schon weit gediehen war und in Berlin längst die Bau- und die Gewerbeakademie existierten, die 1879 zur Königlich Technischen Hochschule Charlottenburg (Berlin) zusammengelegt wurden.

Nicht jeder Mathematiker konnte mit dieser Situation so gelassen umgehen wie Felix Klein. Er war in der preußischen Rheinprovinz geboren, wirkte aber von 1872 bis 1886 in Bayern und Sachsen, Staaten, die 1866 Gegner von Preußen waren. Dort stichelte er auch gegen die „norddeutschen Mathematiker“. Dieser Name meinte zwar spezifisch die Berliner Mathematiker, spielte aber auf den Norddeutschen Bund an, einen förderativ organisierten Staat, der nach der Auflösung des Deutschen Bundes 1866 gegründet worden war und die Gründung des Deutschen Kaiserreichs für die Gebiete nördlich des Mains vorwegnahm. Zu guter Letzt wirkte Klein aber als preußischer Beamter an der (ehemals hannoverschen) Georg-August-Universität Göttingen und vertrat diese auch im Herrenhaus, der Ersten Kammer des preußischen Landtags.
In gewissem Sinne war die DMV in ihrer Gründungsphase also überstaatlich konzipiert, wobei nach [9, S. 36] der Anteil der ausländischen Mitglieder von 7,8\% im Jahre I89I auf 30,1\% im Jahre 1901 und immerhin $39 \% \mathrm{im}$ Jahre 1913 anstieg. Zudem war sie mit einer nicht ganz einfachen innerstaatlichen Situation konfrontiert, sodass es nicht so überraschend ist, ,dass die DMV relativ spät gegründet wurde" [8, S. 103].

Einige andere nationale mathematische Gesellschaften

Die Niederlande mit der 1778 gegründeten KMG mag man noch als Sonderfall betrachten und die Fälle von Vereinigtem Königreich (LMS, I865) und Frankreich (SMF, 1872) sowohl der staatlichen als auch der wissenschaftlichen Etabliertheit zurechnen. Es gibt aber auch zahlreiche andere Fälle von im Vergleich zur DMV recht frühen Gründungen: 1864 die Moskauer mathematische Gesellschaft, 1890 zum ersten Mal die St. Petersburger mathematische Gesellschaft, 1868 die Suomen matemaattinen yhdistys in Finnland, 1873 die Dansk Matematisk Forening in Dänemark. Bleibt man bei den nordischen Ländern, mag die Gründung der Norsk matematisk forening 1918 im Vergleich dazu etwas spät erscheinen, jedoch war Norwegen erst nach der Auflösung der Personalunion mit Schweden im Jahre 1905 (wieder) ein eigenständiger Staat geworden. Der Fall Finnlands beweist allerdings, dass Eigenstaatlichkeit keine notwendige Bedingung für die Gründung einer nationalen mathematischen Gesellschaft war: 1868 war Finnland ein Großfürstentum innerhalb des russischen Kaiserreiches. Die Polskie Towarzystwo Matematyczne hingegen wurde offiziell erst 1919 gegründet, nach der Unabhängigkeit Polens.

Eine vollständige Liste der Gründungsdaten auch nur der europäischen mathematischen Gesellschaften ist hier nicht angestrebt. Gerade mit Bezug auf die DMV und ihre Situation in einem neu gegründeten Nationalstaat sei aber noch ein Blick auf Italien geworfen, das im Risorgimento ungefähr zeitgleich mit Deutschland seine nationale Frage beantwortete. Wie bereits Erwin Neuenschwander herausgestellt hat [7], waren die politische Einigung und der Aufschwung der Mathematik in Italien durchaus positiv korrelliert: Enrico Betti (1823-1892) beispielsweise, ab 1864 Direktor der Scuola Normale Superiore in Pisa (und Namensgeber der Bettizahlen), hatte 1848 im Ersten italienischen Unabhängigkeitskrieg als Freiwilliger gekämpft. Dennoch: Die UMI wurde, wie bereits erwähnt, erst 1922 gegründet, und zwar als Reaktion auf die erste Generalversammlung des Internationalen Wissenschaftsrates 1919 in Brüssel. Allerdings hatte in Italien bereits zuvor der 1884 gegründete Circolo Matematico di Palermo eine auch im Ausland angesehene Plattform zum wissenschaftlichen Austausch zur Verfügung gestellt. 
Eine Schlussbemerkung über strikte disziplinäre Grenzen hinweg

Dieses Jahr findet die erste gemeinsame Jahrestagung von DMV und der Gesellschaft für angewandte Mathematik und Mechanik statt, welche 1922 aus der DMV ausgegründet wurde. Allerdings ist auch der Ort der Jahrestagung bemerkenswert: Nicht nur, dass bereits 1897 eine Jahrestagung der DMV dort stattfand; dort wurde auch am 5. Oktober |89| der Verein zur Förderung des mathematischen und naturwissenschaftlichen Unterrichts gegründet, der demgemäß in diesem Jahr sein 125-jähriges Jubiläum feiert.

Die Mathematikdidaktik wird bei dieser Berücksichtigung besonderer Jubiläen wohlgemerkt nicht unterschlagen: Zwar findet 2016 bereits die 50. Jahrestagung für Didaktik der Mathematik statt; gegründet wurde die Gesellschaft für Didaktik der Mathematik jedoch erst 1975.

\section{Literatur und Websites}

[1] „Dutch Mathematcial Society, Wiskundig Gennotschap“. Newsletter of the European Mathematical Society 35 (March 2000), 12-14.

[2] Danny Beckers: ,The Royal Dutch Mathematical Society since 1778“. Nieuw Archief voor Wiskunde Ser. 5, 9 , Nr. 2 (2008), 147-149.

[3] Richard Elwes: „The EMS Jubilee: Challenges for the Next 25 Years". Newsletter of the European Mathematical Society 98 (December 2015), 3-4.

[4] Dietrich von Engelhardt: „Die Geschichte der GDNÄ“ http://www.gdnae.de/wp-content/uploads/2015/0I/Website_ Geschichte.pdf abgefragt am 3I. I. 2016

[5] Pavel Exner: „Message from the President“ I/2016. http:// www.euro-math-soc.eu/news/16/01/4/message-president abgefragt am 3I. I. 2016

[6] Hélène Gispert, Renate Tobies: „A comparative study of the French and German Mathematical Societies before 1914“. In
L'Europe mathématique - Mythes, histoires, identités. Mathematical Europe - Myths, History, Identity. Hrsg. v. Catherine Goldstein, Jeremy Gray, Jim Ritter. Paris: Éditions de la Maison des Sciences de l'Homme 1996, 409-430.

[7] Erwin Neuenschwander: „Der Aufschwung der italienischen Mathematik zur Zeit der politischen Einigung Italiens und seine Auswirkungen auf Deutschland“'. Symposia Mathematica 27 (1986), 213-237.

[8] Gert Schubring: „120 Jahre Deutsche MathematikerVereinigung: Neue Ergebnisse zu ihrer Geschichte“. Mitteilungen der DMV 18 Heft 2 (2010), 103-108.

[9] Renate Tobies: „I25 Jahre DMV“. Programme Book der Jahrestagung der Deutschen Mathematiker-Vereinigung 2015, Hamburg 2I.-25. 9. 2015. Hamburg: Verein zur Ausrichtung von Tagungen am Fachbereich Mathematik der Universität Hamburg e. V. 2015, 3I-37.

[10] —: „Hermann Schubert, die DMV und Hamburg“. Programme Book der Jahrestagung der Deutschen Mathematiker-Vereinigung 2015, Hamburg 21.-25.9.2015. Hamburg: Verein zur Ausrichtung von Tagungen am Fachbereich Mathematik der Universität Hamburg e. V. 2015, 5I-53.

[II] https://dmv.mathematik.de/index.php/88-dmv/geschichte/ 482-geschichte-der-dmv abgefragt am 31. I. 2016

[12] https://dmv.mathematik.de/index.php/88-dmv/geschichte/ 493-publikationen-zur-geschichte-der-dmv abgefragt am 31. 1.2016

[13] http://www.mathunion.org/general/history/ abgefragt am 31. 1.2016

[14] http://www.hs.uni-hamburg.de/DE/GNT/hh/lfi/mathg-hh. htm abgefragt am 31. I. 2016

Prof. Dr. Peter Ullrich, Universität Koblenz-Landau, Campus Koblenz, Mathematisches Institut, Universitätsstraße I, 56070 Koblenz. ullrich@uni-koblenz.de

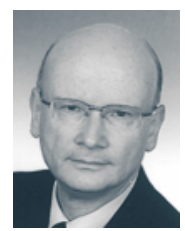

Jahrgang 1957, Studium der Mathematik und Physik in Bielefeld und Münster, Promotion 1985, Habilitation 1992 in Münster, danach akademische Positionen an den Universitäten in Münster, Gießen, Augsburg und Siegen, seit 2005 Professor für Mathematik und ihre Didaktik an der Universität Koblenz-Landau, Vorsitzender der Fachgruppe „Geschichte der Mathematik" der DMV. 